\title{
Affine-by-Statement Transformations of Imperfectly Nested Loops
}

\author{
Jingling Xue ${ }^{\dagger}$ \\ Department of Mathematics, Statistics and Computing Science \\ University of New England, Armidale 2351, Australia
}

\begin{abstract}
A majority of loop restructuring techniques developed so far assume that loops are perfectly nested. The unimodular approach unifies three individual transformations - loop interchange, skewing and reversal - but is still limited to perfect loop nests. This paper outlines a framework that enables the use of unimodular transformations to restructure imperfect loop nests. The concepts previously used for perfect loop nests, such as iteration vector, iteration space and lexicographic order, are generalised to characterise imperfect loop nests. Multiple unimodular transformations are allowed, one on each statement in the loop nests. A code generation algorithm is developed that produces the transformed code by scanning a disjunction of conjunctive affine constraints.
\end{abstract}

\section{Introduction}

A recent study [7] shows that a majority of the loops in scientific code are imperfectly nested, and a majority of the performance-increasing techniques developed in the past two decades assume that loops are perfectly nested. The unimodular approach $[2,3,5,8]$ unifies loop interchange, skewing and reversal, but is limited to perfect loop nests only.

There are two approachs to handling imperfect loop nests. One approach converts imperfect to perfect loop nests and then applies the unimodular approach. Techniques used include loop distribution, loop fusion, Abu-Sufah's nonbasic-to-basic transformation [1], and recently, scalar forward substitution followed by loop distribution [7]. The other approach, which is also the approach taken in this paper, aims at some extensions of the unimodular approach. In [4], matrices are used to model such transformations as loop distribution and loop fusion. In [6], unimodular transformations are generalised to schedules so that a much broader set

\footnotetext{
${ }^{\dagger}$ This work is supported by an Australian Research Council Grant A49600987.
}

of transformations (including, for example, loop distribution and loop fusion) can be represented. A code generation algorithm for implementing schedules was also developed. However, the work was not geared specifically for loop restructuring.

This paper outlines a framework for using unimodular matrices to restructure imperfect loop nests. The concepts previously used for perfect loop nests, such as iteration vector, iteration space and lexicographic order, are generalised to characterise imperfect loop nests (Section 3). We show how to restructure an imperfect loop nest by applying multiple unimodular transformations - possibly with additive constant vectors - one on each statement of the loop nest (Section 4). The paper title is so chosen, partly because these transformations are known as affine transformations and partly because every statement in the loop nest can be transformed by an individual affine transformation. A code generation method is given that produces a possibly imperfect loop nest to scan an iteration space (Section 5). This method subsumes those for perfect loop nests $[2,5]$ while retaining the philosophy of generating code by scanning affine constraints.

\section{Notation and Terminology}

In this preliminary study, the class of loop nests is restricted to those specified by the simple grammar:

$$
\mathbb{L} \rightarrow \mathbb{L} \mathbb{L} \mid \text { do-loop } \mathbb{L} \mid \text { statement }
$$

where nonterminal $\mathbb{L}$ represents a loop nest, terminal do - loop represents a DO loop, and terminal s tatement represents an atomic statement. An atomic statement is a code segment that is transformed as a basic unit. In the context of loop transformations, an atomic statement is usually a single assignment statement, possibly guarded with some conditionals.

A loop nest is perfect if all statements are inside the innermost loop of the loop nest and imperfect otherwise. All loops are assumed to have unity loop strides. 
Our illustrating example is:

$$
\begin{aligned}
& L_{1} \quad \text { do } i=1, n \\
& L_{2,1} \quad \text { do } j=1, n \\
& S_{1} \quad a(i, j)=a(i-1, j)+a(i, j-1) * b(i-1) \\
& L_{2,2} \quad \text { do } k=1, i \\
& S_{2} \quad b(i)=b(i)+a(k, i-k+1)
\end{aligned}
$$

The notation $a \# b$ indicates the concatenation of two vectors $a$ and $b$, As examples, $(4,2) \#(3)=(4,2,3)$, and $(1) \#()=(1)$, where () denotes the null vector.

\section{Modeling Imperfect Loop Nests}

This section extends the concepts for perfect loop nests, such as iteration vector, iteration space and lexicographic order, to characterise imperfect loop nests.

\subsection{Iteration Vector of a Statement}

An imperfect loop nest contains statements that are imperfectly nested in loops of varying depths. To identify an instance of such a statement by an iteration vector, we must include in the iteration vector not only the values of surrounding loop variables but also the values indicating the lexical order of the statement.

The following procedure, when called with arguments $\mathbb{L}$ and ( ), will return the iteration vector $V$ for each of the statements in a loop nest $\mathbb{L}$.

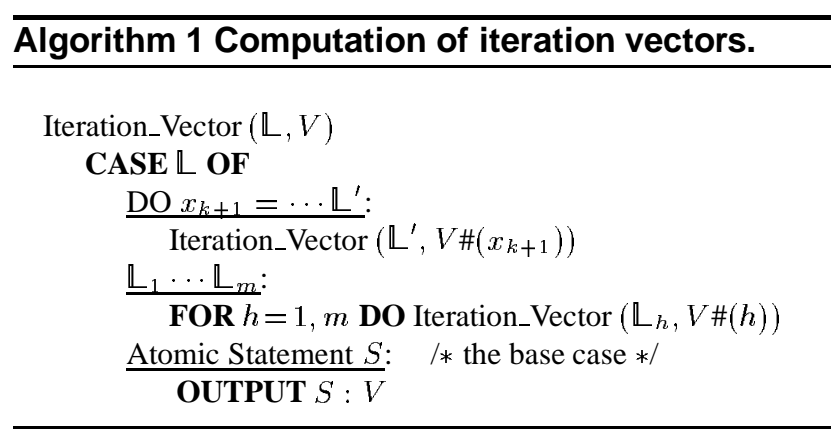

In this and Algorithms 2, it is assumed that $\mathbb{L}$, if it is an atomic statement, will match the $\underline{\mathbb{L}}_{1}, \cdots \mathbb{L}_{m}$ (with $m=1$ ) before it matches the Atomic Statement $S$.

The iteration vectors for the program (1) are:

$$
\begin{aligned}
& S_{1}:(\mathrm{i}, 1, \mathrm{j}, 1) \\
& S_{2}:(\mathrm{i}, 2, \mathrm{k}, 1)
\end{aligned}
$$

The iteration vectors for different statements satisfy two properties: (1) The $i$-th entries, if defined, are either all loop entries or all statement entries. (2) The last entry of an iteration vector is a statement entry.

The definition of iteration vector is consistent with that for perfect loop nests. In the case of an $n$-deep perfect loop nest, the iteration vector for the $i$-th statement $S_{i}$ in the loop body has the form $\left(x_{1}, \cdots, x_{n}, i\right)$, where the first $n$ entries are loop entries. If all statements inside the innermost loop are atomic, each iteration of the loop body can be identified by the iteration vector $\left(x_{1}, \cdots, x_{n}\right)$, as previously.

\subsection{Statement Space of a Statement}

An iteration vector may have both loop and statement entries. Let $\operatorname{loop}(x)$ be a boolean function such that $\operatorname{loop}(x)$ is true if and only if $x$ is a loop entry. The statement space, $I_{S}$, of a statement $S$ consists of all the iterations of the statement, or formally:

$$
I_{S}=\left\{\left(x_{1}, \cdots, x_{S}\right) \mid \forall i: \operatorname{loop}\left(x_{i}\right) \Longrightarrow L_{i} \leq x_{i} \leq U_{i}\right\}
$$

where

- $\left(x_{1}, x_{2}, \cdots, x_{S}\right)$ is the iteration vector of $S$, and

- If $x_{i}$ is a loop entry, then "DO $x_{i}=L_{i}, U_{i}$ " is the $i$-th enclosing loop of $S$.

The following procedure, when called with arguments $S$, () and \{\} , will return the statement space $\{V \mid P\}$ for each of the statements in a loop nest $\mathbb{L}$.

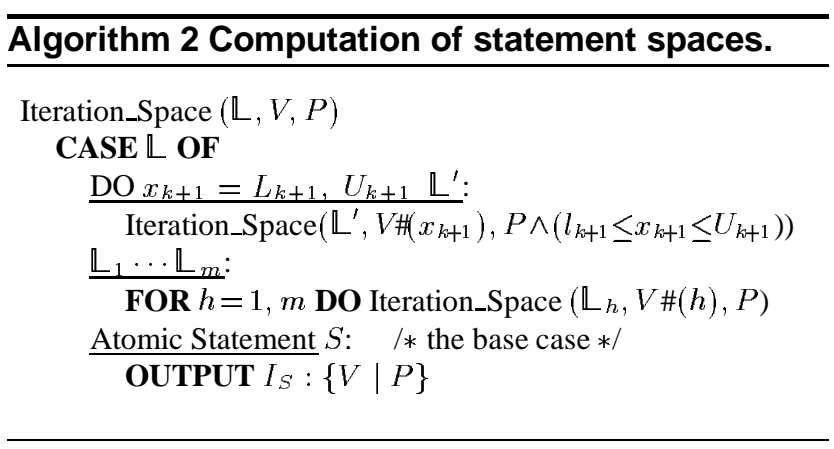

The statement spaces for (1) are as follows:

$$
\begin{aligned}
& I_{S_{1}}:\{(i, 1, j, 1) \mid 1 \leq i, j \leq n\} \\
& I_{S_{2}}:\{(i, 2, k, 1) \mid 1 \leq i \leq n, 1 \leq k \leq i\}
\end{aligned}
$$

A statement spaces is always a convex polyhedron.

\subsection{Iteration Space of a Loop Nest}

The iteration space for a loop nest is the union of all statement spaces in the loop nest. Note that different statement spaces may have different dimensions. This implies that the iteration vectors in an iteration space may have incompatible lengths. In addition, an iteration space is not a convex polyhedron, in general. 


\subsection{Lexicographic Order}

The lexicographic order "less than" $\prec$ on two iteration vectors $x=\left(x_{1}, \cdots, x_{n}\right)$ and $y=\left(y_{1}, \cdots, y_{m}\right)$ is defined inductively as follows:

$$
\begin{aligned}
n=1 \vee m=1: & \left(x_{1}, \cdots, x_{n}\right) \prec\left(y_{1}, \cdots, y_{m}\right) \\
n>1 \wedge m>1: & \left.\Longleftrightarrow x_{1}, \ldots, x_{n}\right) \prec\left(y_{1}, \ldots, y_{m}\right) \\
& \Longleftrightarrow\left\{\begin{array}{l}
x_{1}<y_{1} \text { or } \\
x_{1}=y_{1} \wedge\left(x_{2}, \ldots, x_{n}\right) \prec\left(y_{2}, \ldots, y_{m}\right)
\end{array}\right.
\end{aligned}
$$

According to the definition of iteration vector, there do not exist two iteration vectors such that one is the prefix of the other. Therefore, $\prec$ is well-defined.

Theorem 1 In a sequential execution, the iterations of a loop nest are executed according to the lexicographic order of their iteration vectors.

\section{Unimodular Transformations}

The iteration space of a loop nest is a union of statement spaces, where each statement space is a convex polyhedron. Loop transformations are cast as unimodular transformations from statement spaces to statement spaces. Such a framework allows a restructuring compiler to apply several transformations at one step, each on a distinct statement space.

Let $x$ be the iteration vector of a statement $S$. The notation $x_{L}$ denotes the vector obtained from the $x$ with all its statement entries removed. Then the statement space of a statement $S$ can be specified as a convex polyhedron:

$$
I_{S}=\left\{x \mid A x_{L} \leq b\right\}
$$

For example, the following statement space

$$
I_{S_{1}}=\{(i, 1) \mid 1 \leq i \leq n\}
$$

can be put into the matrix form:

$$
I_{S_{1}}=\left\{(i, 1) \mid\left[\begin{array}{r}
-1 \\
1
\end{array}\right][i] \leq\left[\begin{array}{r}
-1 \\
n
\end{array}\right]\right\}
$$

where $x=(i, 1)$ and $x_{L}=(i)$.

To ensure that the statement space of $S$ remains to be a convex polyhedron in the restructured code, we require that $S$ be enclosed by the same number of loops before and after the loop nest is restructured. Let $y\left(I_{S}^{\prime}\right)$ be the iteration vector (statement space) of $S$ in the restructured code. We use two separate mappings to transform $I_{S}$ to $I_{S}^{\prime}$ : one defines the iteration vector $y$ (or essentially, the values of statement entries in $y$ ), and the other is an affine transformation $\left(T_{S}, \alpha_{S}\right)$, where $T_{S}$ is unimodular and $\alpha_{S}$ is an additive constant vector, such that $T_{S} x_{L}+\alpha_{S}=y_{L}$. The transformed statement space $I_{S}^{\prime}$ is a convex polyhedron:

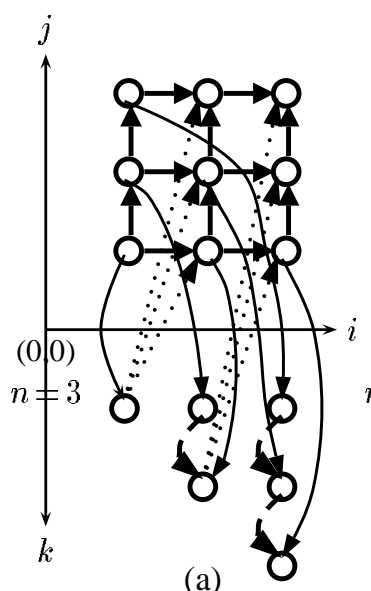

(a)

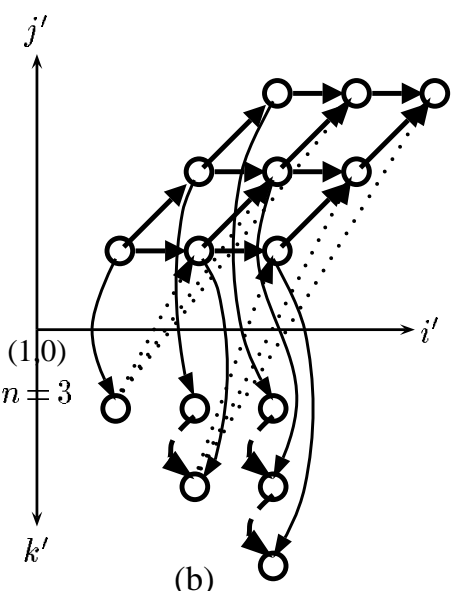

(b)
Figure 1. (a) The iteration space of (1). The horizontal arrows represent $(1,0)$, the vertical arrows $(0,1)$, the dotted arrows $(1)$, the curved solid arrows $(<)$ and the dashed arrows $(0,1)$. (b) The transformed iteration space.

$$
I_{S}^{\prime}=\left\{y \mid A T_{S}^{-1}\left(y_{L}-\alpha_{S}\right) \leq b\right\}
$$

When $\alpha_{S}=0$, the unimodular transformation $T_{S}$ describes all combinations of loop interchange, skewing and reversal. By considering $S$ and its surrounding loops in isolation as a perfect loop nest, we are essentially transforming this perfect loop nest using the affine transformation $\left(T_{S}, \alpha_{S}\right)$. We must replace the old loop variables $x_{L}$ in the array subscripts of statement $S$ by the new ones $y_{L}-$ a simple process because $T_{S} x_{L}+\alpha_{S}=y_{L}$, where $T_{S}$ is invertible.

For example, consider the imperfect loop nest (1). Figure 1(b) shows its iteration space $I_{S_{1}} \cup I_{S_{2}}$ given in (2), annotated with the following data dependencies:

- The dependence from $a(i, j)$ to $a(i-1, j)$ has distance vector $(1,0)$.

- The dependence from $a(i, j)$ to $a(i, j-1)$ has distance vector $(0,1)$.

- The dependence from $a(i, j)$ to $a(k, i-k+1)$ has direction vector $(\leq)$.

- The dependence from the LHS $b(i)$ of $S_{2}$ to the RHS $b(i)$ of the same statement has distance vector $(0,1)$.

- The dependence from the LHS $b(i)$ of $S_{2}$ to $b(i-1)$ has distance vector (1).

Since there is a cycle of data dependences between $S_{1}$ and $S_{2}$, it is illegal to use loop distribution to convert the program into perfectly nested loops. This means that the existing unimodular approach cannot be used. 
In our framework, we may restructure this loop nest as follows (Figure 1(b)):

- Apply a loop skewing followed by a loop interchange, modeled by the transformation:

$$
T_{S_{1}}=\left[\begin{array}{ll}
1 & 1 \\
0 & 1
\end{array}\right], \quad T_{S_{1}}\left[\begin{array}{l}
i \\
j
\end{array}\right]=\left[\begin{array}{l}
i^{\prime} \\
j^{\prime}
\end{array}\right]
$$

to the $i$ and $j$ loops transforms $I_{S_{1}}$ to:

$$
I_{S_{1}}^{\prime}=\left\{\left(i^{\prime}, 1, j^{\prime}, 1\right) \mid 1 \leq i^{\prime}-j^{\prime} \leq n, 1 \leq j^{\prime} \leq n\right\}
$$

- Apply the index shifting transformation

$$
T_{S_{2}}=\left[\begin{array}{ll}
1 & 0 \\
0 & 1
\end{array}\right], T_{S_{2}}\left[\begin{array}{l}
i \\
j
\end{array}\right]+\left[\begin{array}{l}
1 \\
0
\end{array}\right]=\left[\begin{array}{l}
i^{\prime} \\
j^{\prime}
\end{array}\right]
$$

to the $i$ and $k$ loops transforms $I_{S_{2}}$ to:

$$
I_{S_{2}}^{\prime}=\left\{\left(i^{\prime}, 2, k^{\prime}, 1\right) \mid 2 \leq i^{\prime} \leq n+1,1 \leq k^{\prime} \leq i^{\prime}\right\}
$$

Our code generation method will generate the following code to scan $I_{S_{1}}^{\prime} \cup I_{S_{2}}^{\prime}$ lexicographically:

$$
\begin{aligned}
& \text { do } i^{\prime}=2, n+1 \\
& \text { do } j^{\prime}=\max \left(1, i^{\prime}-n\right), \min \left(i^{\prime}-1, n\right) / * \text { parallelisable } * / \\
& \quad a\left(i^{\prime}-j^{\prime}, j^{\prime}\right)=a\left(i^{\prime}-j^{\prime}-1, j^{\prime}\right)+a\left(i^{\prime}-j^{\prime}, j^{\prime}-1\right) * b\left(i^{\prime}-j^{\prime}-1\right) \\
& \text { do } k^{\prime}=1, i^{\prime} \\
& \quad b\left(i^{\prime}\right)=b\left(i^{\prime}\right)+a\left(k^{\prime}, i^{\prime}-k^{\prime}+1\right) \\
& \text { do } i^{\prime}=n+2,2 n \\
& \text { do } j^{\prime}=i^{\prime}-n, n / * \text { parallelisable } * / \\
& \quad a\left(i^{\prime}-j^{\prime}, j^{\prime}\right)=a\left(i^{\prime}-j^{\prime}-1, j^{\prime}\right)+a\left(i^{\prime}-j^{\prime}, j^{\prime}-1\right) * b\left(i^{\prime}-j^{\prime}-1\right)
\end{aligned}
$$

The two transformations we have applied are legal because the original five dependencies are mapped into $(1,0),(1,1)$, $(=),(0,1)$ and $(<)$, which are all lexicographically positive. The two $j^{\prime}$ loops can be run in parallel since they do not carry any dependencies.

We are currently studying how to systematically choose a combination of multiple transformations to exploit collectively the available parallelism in a loop nest, and the required dependence test for legality.

\section{Code Generation}

Let $S_{1}, \cdots, S_{m}$ be the statements in a loop nest. Let their statement spaces $I_{S_{1}}, \cdots, I_{S_{m}}$ be transformed by some unimodular transformations. The transformed statement spaces $I_{S_{1}}^{\prime}, \cdots, I_{S_{m}}^{\prime}$ are all convex polyhedra. In the transformed iteration space $\bigcup_{k} I_{S_{k}}^{\prime}$, the iterations are executed lexicographically with respect to that space. We need to generate code to iterate over such a space in lexicographic order.

To construct a loop nest to scan an iteration space, our code generation procedure Code_Gen $(I)$ given below proceeds in three steps:

\section{Algorithm 3 Code generation algorithm.}

Code_Gen $(I)$

I* $I$ is an iteration space as a set of statement spaces $* /$

Step 1: Explicitly_Defined $(I)$

Step 2: (a) Create a tree with Root and $T$ set below:

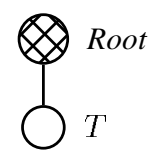

(b) Tree_Gen $(I, T, 1)$

Step 3: Loop_Gen(Root)

Step 1: Explicitly_Defined(I) examines every statement space in the iteration space $I$ and introduces extra constraints, if necessary, to ensure that every loop variable in every statement space is explicitly bounded from both below and above by some constraints. For example, all constraints in the $I_{S_{1}}^{\prime}$ of (3) are loop bounds of $j^{\prime}$. Using the Fourier-Motzkin elimination, we obtain the loop bounds of $i^{\prime}: 2 \leq i^{\prime} \leq 2 n$. So in the first step, Explicitly_Defined will modify $I_{S_{1}}^{\prime}$ to:

$I_{S_{1}}^{\prime}=\left\{\left(i^{\prime}, 1, j^{\prime}, 1\right) \mid 2 \leq i^{\prime} \leq 2 n, 1 \leq i^{\prime}-j^{\prime} \leq n, 1 \leq j^{\prime} \leq n\right\}$

This step ensures that the bounds of all loop variables are well-defined.

Step 2: Starting with the initial tree in Step (a), Tree_Gen $(I, T, 1)$, written as Algorithm 4 and called in (b), builds a tree representation for the iteration space $I$, starting from the first entry of an iteration vector.

Step 3: Loop_Gen(Root) generates the code by applying a preorder traversal of the tree in Step 2.

\section{Conclusion}

This paper outlined a matrix transformation framework for restructuring imperfect loop nests. The concepts previously used for perfect loop nests, such as iteration vector, iteration space and lexicographic order, were generalised to characterise imperfect loop nests. It was shown how multiple affine transformations can be supported, one on each statement of a loop nest. A code generation algorithm was given that produces code for an iteration space by scanning a disjunction of conjunctive affine constraints. This algorithm extends the existing ones while retaining their philosophy of generating code by scanning affine constraints. 


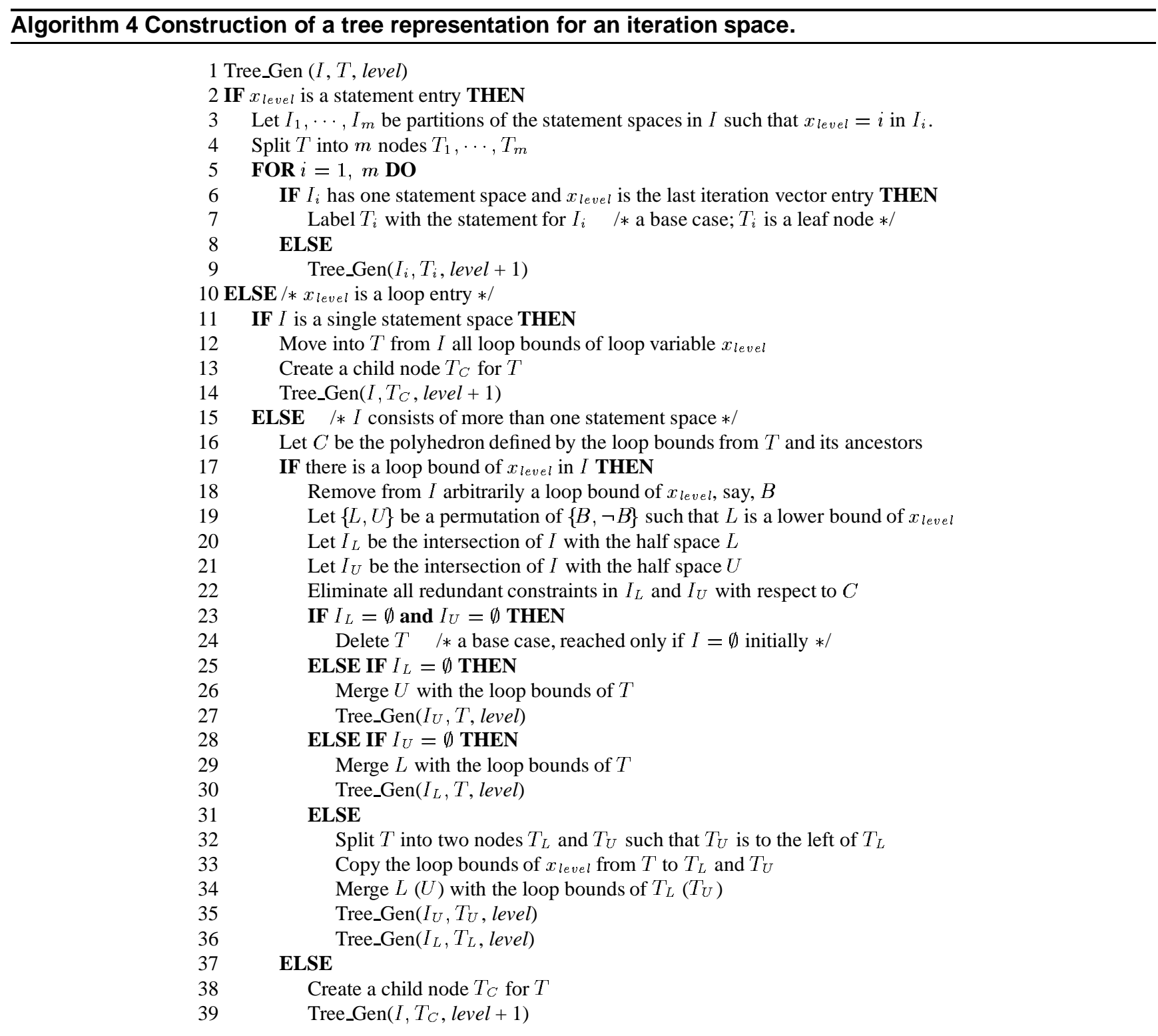

\section{References}

[1] W. Abu-Sufah. Improving the Performance of Virtual Memory Computers. PhD thesis, University of Illinois at UrbanaChampaign, Nov. 1978.

[2] C. Ancourt and F. Irigoin. Scanning polyhedra with DO loops. In Proc. Third ACM SIGPLAN Symp. on Principles \& Practice of Parallel Programming (PPoPP), pages 39-50. ACM Press, Apr. 1991.

[3] U. Banerjee. Unimodular transformations of double loops. In A. Nicolau, D. Gelernter, T. Gross, and D. Padua, editors, Advances in Languages and Compilers for Parallel Processing, chapter 10, pages 192-219. MIT Press, 1991.

[4] D. R. Chesney and B. H. C. Cheng. Generalising the unimodular approach. In L. M. Ni, editor, 1994 Int. Conf. on Parallel and Distributed Systems, pages 398-403, Taiwan, 1994. IEEE Computer Society Press.

[5] J.-F. Collard, T. Risset, and P. Feautrier. Construction of DO loops from systems of affine constraints. Technical Report 93-15, Ecole Normale Supérieure de Lyon, May. 1993.

[6] W. Kelly and W. Pugh. A framework for unifying reordering transformations. Technical Report CS-TR-2995, Department of Computer Science, University of Maryland, Aug. 1993.

[7] R. Sass and M. Mutka. Enabling unimodular transformations. In Proc. of Supercomputing '94. IEEE Computer Society Press, 1994.

[8] M. Wolf and M. Lam. A loop transformation theory and an algorithm to maximize parallelism. IEEE Trans. on Parallel and Distributed Systems, 2(4):452-471, Oct. 1991. 